\title{
Two confirmed class I very low-mass objects in Taurus
}

\author{
C. Dang-Duc ${ }^{1,3}$, N. Phan-Bao ${ }^{1,2}$, and D. T. Dao-Van ${ }^{1}$ \\ ${ }^{1}$ Department of Physics, HCM International University-VNU, Block 6, Linh Trung Ward, Thu Duc District, HCM city, Viet Nam \\ e-mail: pbngoc@hcmiu.edu.vn \\ 2 Institute of Astronomy and Astrophysics, Academia Sinica, PO Box 23-141, 106 Taipei, Taiwan, ROC \\ 3 Faculty of Physics and Engineering Physics, HCM University of Science-VNU, 227 Nguyen Van Cu Street, District 5, \\ HCM city, Viet Nam
}

Received 10 December 2015 / Accepted 10 February 2016

\begin{abstract}
Context. [GKH94] 41 and IRAS 04191+1523B were previously identified to be proto-brown dwarf candidates in Taurus. [GKH94] 41 was classified to be a class I object. The dereddened spectral energy distribution of the source was later found to be suggestive of a class II object. IRAS $04191+1523$ B is a class I object that is the secondary component of a binary.

Aims. We determine the evolutionary stage of [GKH94] 41 and estimate the final masses of the two proto-brown dwarf candidates. Methods. We used archive millimeter observations to produce continuum maps and collected data from the literature to construct the spectral energy distribution of the targets.

Results. Our continuum maps revealed that both [GKH94] 41 and IRAS 04191+1523B are surrounded by envelopes. This provides direct evidence that [GKH94] 41 is a class I object, not class II, as previously classified. For IRAS 04191+1523B, our continuum map spatially resolved the binary. Our estimated final masses are below $49_{-27}^{+56} M_{\mathrm{J}}$ and $75_{-26}^{+40} M_{\mathrm{J}}$ for [GKH94] 41 and IRAS 04191+1523B, respectively. This indicates that both sources will likely become brown dwarfs or very low-mass stars. Therefore, [GKH94] 41 and IRAS $04191+1523 \mathrm{~B}$ are two new confirmed class I very low-mass objects. Their existence also supports the scenario that brown dwarfs have the same formation stages as low-mass stars.
\end{abstract}

Key words. brown dwarfs - stars: formation - stars: protostars - techniques: interferometric

\section{Introduction}

In 1995, the first brown dwarfs (BDs, 13-75 $M_{\mathrm{J}}$ ) were discovered (e.g., Rebolo et al. 1995). Up to date, thousands of BDs have been identified in star-forming regions and in the solar neighborhood. The coolest BDs (e.g., Y dwarfs) have temperatures as cool as the human body. BDs are thought to form by different mechanisms. Two major models have been proposed for the BD formation. In the starlike models, very low-mass (VLM) cores are produced by turbulent fragmentation (Padoan \& Nordlund 2004) or gravitational fragmentation (Bonnell et al. 2008) of molecular clouds. In the ejection models, BDs are simply VLM embryos ejected from unstable multiple protostellar systems by dynamical interaction (e.g., Bate et al. 2002).

Observations of statistical properties of BDs such as initial mass function, velocity dispersion, multiplicity, accretion disks, and jets (see Luhman 2012, and references therein) have indicated that these statistical properties of BDs form a continuum with those of low-mass stars (see Whitworth et al. 2007, and references therein). This therefore strongly supports starlike models for BD formation. Recent observations of molecular outflows in class II BDs and VLM stars (Phan-Bao et al. 2008, 2011, 2014) have shown that the molecular outflow process occurs in these VLM objects as a scaled-down version of that seen in low-mass stars. The authors also suggested that the associated accretion process is possibly episodic and has a very low accretion rate. This may prevent a VLM core that might be directly produced by a process of turbulent fragmentation (Padoan \& Nordlund 2004) to accrete enough material to become a star. More observations of proto-BDs at earlier classes are clearly needed to confirm these results.

So far, a few candidates of class $0 / \mathrm{I}$ proto-BDs have been identified (e.g., Bourke et al. 2006; Lee et al. 2013; Palau et al. 2014; Morata et al. 2015). Of these candidates, only IC 348SMM2E and L328-IRS are class 0 objects with estimated final masses below the hydrogen-burning limit (Lee et al. 2013; Palau et al. 2014). André et al. (2012) identified a pre-BD with an estimated final mass to be substellar. These sources are rare benchmarks of BDs in the protostellar phase on which their origin can be studied. Their existence has also demonstrated that BDs and low-mass stars share the same manner of formation process. Here, we report our study of two class I proto-BD candidates in Taurus, [GKH94] 41 and IRAS 04191+1523B.

\section{Targets}

We selected [GKH94] 41 (2MASS J04194657+2712552) and IRAS 04191+1523B (2MASS J04220007+1530248) in the list of 352 members of Taurus from Luhman et al. (2010). Within the class I objects, they are class I proto-BD candidates with spectral types later than M6.

[GKH94] 41 was primarily identified as an embedded protostar in Taurus based on its very red near-infrared colors and extended appearance (Gomez et al. 1994). Its estimated spectral type of M7.5 \pm 1.5 implies that the central object is substellar (Luhman et al. 2009). The flat spectral energy distribution (SED) of [GKH94] 41 in the infrared region suggested the source to be in the class I or very young class II evolutionary 
stage (Luhman et al. 2009). Its dereddened SED was later found to be suggestive of a class II BD surrounded by a disk and not by an envelope (Furlan et al. 2011).

IRAS $04191+1523 \mathrm{~B}$ is the secondary component of a $6 . ' 1$ binary (Duchêne et al. 2004). The spectral types that were estimated based on luminosity are K6-M3.5 and M6-M8 for IRAS 04191+1523A (2MASS J04220043+1530212) and IRAS 04191+1523B, respectively (Luhman et al. 2010). IRAS 04191+1523B is a class I object (Luhman et al. 2010). Its spectral type in the range of M6-M8 indicates that the central source is a substellar or VLM object. In the following sections, we consider IRAS $04191+1523 \mathrm{~B}$ to be an M7.0 \pm 1.0 , which is an average spectral type of M6 and M8.

\section{Millimeter continuum observations}

We searched the Combined Array for Research in Millimeterwave Astronomy (CARMA) data archive for observations of [GKH94] 41 and IRAS 04191+1523B. Both targets were observed on 2012 December 25 with CARMA (Project C1013). All six 10.4-meter, nine 6.1-m antennas were operated in the $\mathrm{C}$ configuration at about $102 \mathrm{GHz}$ (or $\sim 2.9 \mathrm{~mm}$ ). Zenith opacities at $102 \mathrm{GHz}$ were in the range of $0.24-0.38$. The CARMA correlator was set up in the continuum mode of sixteen $500 \mathrm{MHz}$ wide bands with 39 channels per band. The quasars 3C 111 and $0510+180$ were observed for gain, and 3C 84 for passband and flux calibration. The uncertainty in the absolute flux calibration is about $15 \%$. We used the MIRIAD package adapted for the CARMA to reduce the continuum data. The synthesized beam sizes are about $2.19^{\prime \prime} \times 1.53^{\prime \prime}$ and $1.90^{\prime \prime} \times 1.59^{\prime \prime}$ (natural weighting) for [GKH94] 41 and IRAS 04191+1523B, respectively. The primary FWHM beam is about $81^{\prime \prime}$ at the observed frequency.

\section{Results and discussion}

The continuum emission from both targets is clearly detected. Figure 1 shows the continuum map of [GKH94] 41. The position of the emission peak coincides with the 2MASS near-infrared position of the source. This indicates that the continuum emission is associated with [GKH94] 41. The rms noise measured near the map center is about $0.2 \mathrm{mJy}$ beam $^{-1}$. The integrated flux from [GKH94] 41 is measured from a Gaussian fitting to be $2.5 \pm 0.2 \mathrm{mJy}$. The deconvolved sizes of the emission that can be estimated from the 2D Gaussian fitting are listed in Table 1. The deconvolved major axis of the emission region is about 2.9" or $\sim 400 \mathrm{AU}$ in length at a distance of $140 \mathrm{pc}$ (see Table 1), comparable to the typical size of disks associated with class I low-mass objects (200-400 AU, e.g., Lee 2011, Harsono et al. 2014). However, since we would expect that the disk size decreases with the mass of the central object and since the typical size of disks associated with class II BDs is significantly smaller (140-280 AU in diameter, e.g., Ricci et al. 2014), our large measured size suggests that the compact structure associated with [GKH94] 41 is dominated by an envelope and that this object is in the class I evolutionary stage. The bolometric temperature estimated for [GKH94] 41 is $\sim 460 \mathrm{~K}$, further supporting that the source is a late class I object (300-650 K, Enoch et al. 2009). We note that Furlan et al. (2011) argued that the dereddened SED of the source is similar to the SED of a typical T Tauri star with silicate emission features at 10 and $20 \mu \mathrm{m}$ and no ice absorption features. The authors then concluded that [GKH94] 41 is a heavily extinguished class II object surrounded by a disk and not by an envelope. A possible explanation for

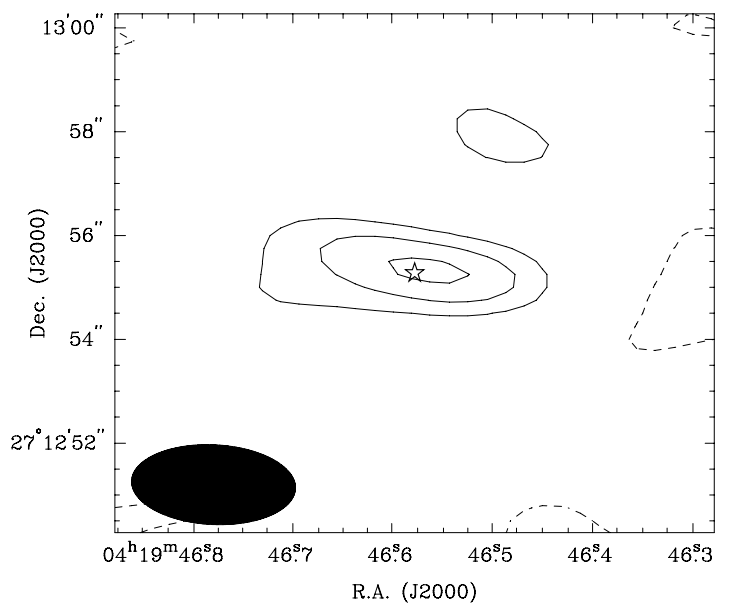

Fig. 1. Continuum map at $2.9 \mathrm{~mm}$ of [GKH94] 41. The star symbol indicates the 2MASS near-infrared position of [GKH94] 41. The contours are $-3,3,5$, and 7 times the rms of $0.2 \mathrm{mJy}^{\text {beam }}{ }^{-1}$. The synthesized beam is indicated in the bottom left corner.

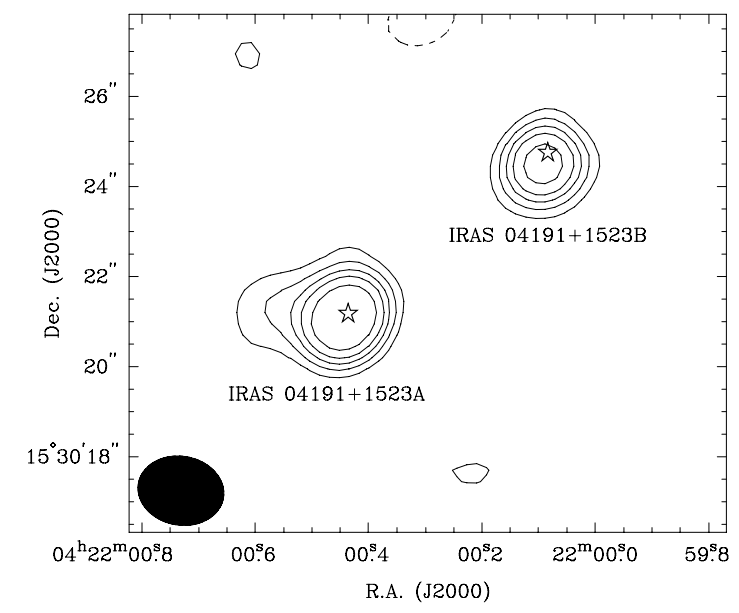

Fig. 2. Continuum map at $2.9 \mathrm{~mm}$ of IRAS $04191+1523 \mathrm{~A}$ and IRAS $04191+1523 \mathrm{~B}$. Two components are spatially resolved with a separation of 6.' 1 . The star symbols represent the 2MASS near-infrared positions of IRAS $04191+1523 \mathrm{~A}$ and IRAS $04191+1523 \mathrm{~B}$. The contours are $-3,3,5,7,9$, and 12 times the rms of 0.4 mJy beam $^{-1}$. The synthesized beam is indicated in the bottom left corner.

the Furlan et al. (2011) result is that the visual extinction value $\left(A_{\mathrm{V}}=27\right)$ that was used in the dereddening of SED could be overestimated (see Sect. 4.1).

For IRAS 04191+1523B (see Fig. 2) our map spatially resolves the binary into two components. Both components are associated with envelopes. The separation between the two peaks of emission from the envelopes is about 6.' 1 , which is consistent with the previous measurement (Duchêne et al. 2004). The rms noise measured near the map center is about $0.4 \mathrm{mJy}_{\text {beam }}^{-1}$. The integrated fluxes are measured from Gaussian fittings to be $9.5 \pm 0.4 \mathrm{mJy}$ and $5.6 \pm 0.4 \mathrm{mJy}$ for IRAS $04191+1523 \mathrm{~A}$ and IRAS $04191+1523 \mathrm{~B}$, respectively. The deconvolved sizes of the emission from IRAS $04191+1523$ A are listed in Table 1. For IRAS $04191+1523 B$, the Gaussian fitting is unable to deconvolve the emission, which appears to be a point source for the current synthesized beam size.

To determine whether [GKH94] 41 and IRAS 04191+1523B are class I proto-BDs, or in other words, whether these objects finally have substellar masses, we estimate the upper limits to the final masses of the two objects in the following sections. 
C. Dang-Duc et al.: Two class I VLM objects

Table 1. Estimated parameters of the $2.9 \mathrm{~mm}$ continuum sources associated with [GKH94] 41, IRAS 04191+1523A, and IRAS 04191+1523B.

\begin{tabular}{lcccccc}
\hline \hline Source & $\begin{array}{c}\text { Deconvolved } \\
\text { major axis }\left(^{(\prime)}\right.\end{array}$ & $\begin{array}{c}\text { Deconvolved } \\
\text { minor axis }\left({ }^{\prime \prime}\right)\end{array}$ & $\begin{array}{c}\text { PA } \\
\left({ }^{\circ}\right)\end{array}$ & $\begin{array}{c}\text { Peak intensity } \\
(\mathrm{mJy})\end{array}$ & $\begin{array}{c}\text { Flux } \\
(\mathrm{mJy})\end{array}$ & $\begin{array}{c}M_{\text {env }}{ }^{b} \\
\left(M_{\mathrm{J}}\right)\end{array}$ \\
\hline [GKH94] 41 & $2.9 \pm 0.1$ & $0.3 \pm 0.1$ & $83 \pm 7$ & $1.5 \pm 0.2$ & $2.5 \pm 0.2$ & 8 \\
IRAS 04191+1523A & $1.4 \pm 0.1$ & $0.3 \pm 0.1$ & $84 \pm 19$ & $7.0 \pm 1.0$ & $9.5 \pm 0.4$ & 31 \\
IRAS 04191+1523B & $1.8 \pm 0.1^{a}$ & $1.6 \pm 0.1^{a}$ & $-74 \pm 11^{a}$ & $6.0 \pm 0.2$ & $5.6 \pm 0.4$ & 18 \\
\hline
\end{tabular}

Notes. ${ }^{(a)}$ Undeconvolved values; ${ }^{(b)}$ envelope masses (see Sect. 4 for further details).

\section{1. [GKH94] 41: a class I proto-BD}

The mass that is added to the central object should be lower than the mass of material still available in the envelope associated with the central object. The upper limit to the final mass of a stellar object can therefore be estimated from the current mass of the central object and the mass of its envelope.

First, to estimate the current mass of [GKH94] 41, we used the temperature versus mass relation as given in the DUSTY model (Chabrier et al. 2000). For a young object with spectral type M7.5, the temperature is $2795 \mathrm{~K}$ (see Table 8 in Luhman et al. 2003). Because evolutionary models for VLM stars and BDs have high uncertainties at ages younger than $1 \mathrm{Myr}$ (Baraffe et al. 2002), we assumed an age of 1 Myr for [GKH94] 41. The temperature of $2795 \mathrm{~K}$ then corresponds to a mass of $\sim 41 M_{\mathrm{J}}$. If the uncertainty of 1.5 subclasses in the spectral type is taken into account, a possible mass range for [GKH94] 41 is from 14 to $97 M_{\mathrm{J}}$. We also calculated the luminosity of the source itself (without any contribution from its surrounding envelope) to constrain the mass range. We first dereddened the 2MASS $K$-band magnitude $\left(K_{\mathrm{S}}=11.89\right)$ using the extinction law with $R_{\mathrm{V}}=3.1$ (Mathis 1990) and the visual extinction $A_{\mathrm{V}}=4.0$ (Bulger et al. 2014). The $A_{\mathrm{V}}$ value was obtained from the best-fit atmospheric model (see Bulger et al. 2014 and references therein) over the 2MASS $J H K_{S}$ bands. Using the bolometric correction in $K$-band versus spectral type relation as given in Filippazzo et al. (2015), we derived a luminosity of $0.025 L_{\odot}$. Figure 3 shows the luminosity versus temperature diagram for [GKH94] 41 with the theoretical evolutionary models from Chabrier et al. (2000) and Baraffe et al. (1998). The location of [GKH94] 41 in the diagram indicates that the upper limit (including the uncertainties) to its current mass is just above the substellar limit and below $0.09 M_{\odot}$, which is consistent with the upper limit value of $97 M_{\mathrm{J}}$ estimated above. We therefore conclude that the upper limit to the mass of [GKH94] 41 is in the range of 14-97 $M_{\mathrm{J}}$.

Second, we estimated the envelope mass of [GKH94] 41 based on the modeling of a modified blackbody with fluxes from $70 \mu \mathrm{m}$ to $\mathrm{mm}$ wavelengths (Table 2) as used to estimate the envelope mass of IC348-SMM2E (see Palau et al. 2014). We obtain the dust temperature $T_{\mathrm{d}}$, dust emissivity $\beta$, and envelope mass $M_{\text {env }}$ by searching these three parameters minimizing the $\chi^{2}$ in the following ranges: $T_{\mathrm{d}}$ from $10 \mathrm{~K}$ to $40 \mathrm{~K}, \beta$ from 0.1 to 1.8 and $M_{\text {env }}$ from $1 M_{\mathrm{J}}$ to $60 M_{\mathrm{J}}$. We assumed a dust opacity coefficient dependence on wavelength $\lambda$ with $\kappa_{\lambda} \propto \lambda^{-\beta}$, $\kappa_{870 \mu \mathrm{m}}=0.0175 \mathrm{~cm}^{2} \mathrm{~g}^{-1}$ (see Palau et al. 2014 and references therein) and a gas-to-dust ratio of 100 . The best fit (Fig. 4) then gives $T_{\mathrm{d}}=34 \mathrm{~K}, M_{\mathrm{env}}=2 M_{\mathrm{J}}$, and $\beta=0.4$. The obtained dust emissivity index value is significantly lower than the typical value of about 1.4 for class I objects in Taurus (Chandler et al. 1998). This is probably because the best fit is obtained from only three available data points. If we take $\beta=1.4$ and $T_{\mathrm{d}}=34 \mathrm{~K}$, we obtain an envelope mass of $8 M_{\mathrm{J}}$, a factor of 4 higher than the value estimated from the fitting.

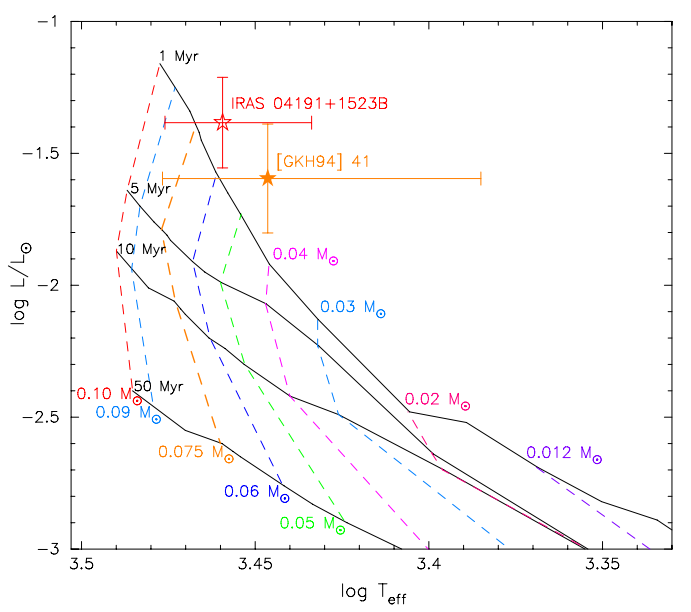

Fig.3. H-R diagram for [GKH94] 41 and IRAS 04191+1523B. Isochrones and mass tracks from the theoretical evolutionary models of Chabrier et al. (2000) for $M \leq 0.075 M_{\odot}$ and Baraffe et al. (1998) for $M>0.075 M_{\odot}$. The solid and open stars represent [GKH94] 41 $(\mathrm{M} 7.5 \pm 1.5)$ and IRAS $04191+1523 \mathrm{~B}(\mathrm{M} 7.0 \pm 1.0)$, respectively. The error bars reflect the uncertainties in the spectral type, 1.5 subclasses for [GKH94] 41 (Luhman et al. 2009) and 1.0 subclass for IRAS 04191+1523B (Luhman et al. 2010).

Table 2. Photometry for [GKH94] 41.

\begin{tabular}{cccc}
\hline \hline $\begin{array}{c}\text { Wavelength } \\
(\mu \mathrm{m})\end{array}$ & $\begin{array}{c}\text { Flux } \\
(\mathrm{mJy})\end{array}$ & $\begin{array}{c}\text { Error } \\
(\mathrm{mJy})\end{array}$ & References \\
\hline 70 & 269 & 5 & 1 \\
160 & 279 & 66 & 1 \\
2940 & 2.5 & 0.2 & this paper \\
\hline
\end{tabular}

References. (1) Bulger et al. (2014).

We therefore take the final mass of [GKH94] 41 to be $49 M_{\mathrm{J}}$, which the sum of the current mass $\left(41 M_{\mathrm{J}}\right)$ and the envelope mass $\left(8 M_{\mathrm{J}}\right)$. If the uncertainty in the spectral type is taken into account, this upper limit will be $49_{-27}^{+56} M_{\mathrm{J}}$. As the outflow process also ejects accreting material and dissipates material in the envelope, the final mass of [GKH94] 41 thus will very likely be below the substellar limit.

\subsection{IRAS 04191+1523B: a class I proto-BD or VLM star}

To estimate the upper limit to the final mass of IRAS $04191+1523 \mathrm{~B}$, we followed the same steps as for [GKH94] 41. A spectral type of M7.0 corresponds to a temperature of $2880 \mathrm{~K}$ (Luhman et al. 2003). Using the DUSTY model for an age of $1 \mathrm{Myr}$, we derive a mass of $\sim 57 M_{\mathrm{J}}$ for the source. If we take the uncertainty of 1.0 subclass in the spectral type into account, a possible mass range is 31-97 $M_{\mathrm{J}}$ for the object. To calculate the luminosity of the source, we used 
Table 3. Photometry for IRAS $04191+1523 \mathrm{AB}$.

\begin{tabular}{cccc}
\hline \hline $\begin{array}{c}\text { Wavelength } \\
(\mu \mathrm{m})\end{array}$ & $\begin{array}{c}\text { Flux } \\
(\mathrm{mJy})\end{array}$ & $\begin{array}{c}\text { Error } \\
(\mathrm{mJy})\end{array}$ & References \\
\hline 70 & 7002 & 13 & 1 \\
160 & 8884 & 232 & 1 \\
450 & 3940 & 220 & 2 \\
850 & 1380 & 20 & 2 \\
1300 & 110 & 7 & 3 \\
2940 & $9.5^{a}$ & $0.4^{a}$ & this paper \\
& $5.6^{b}$ & $0.4^{b}$ & this paper \\
\hline
\end{tabular}

Notes. ${ }^{(a)}$ The flux and its error for component IRAS 04191+1523A. (b) The flux and its error for component IRAS 04191+1523B.

References. (1) Bulger et al. (2014); (2) Francesco et al. (2008); (3) Motte \& André (2001).

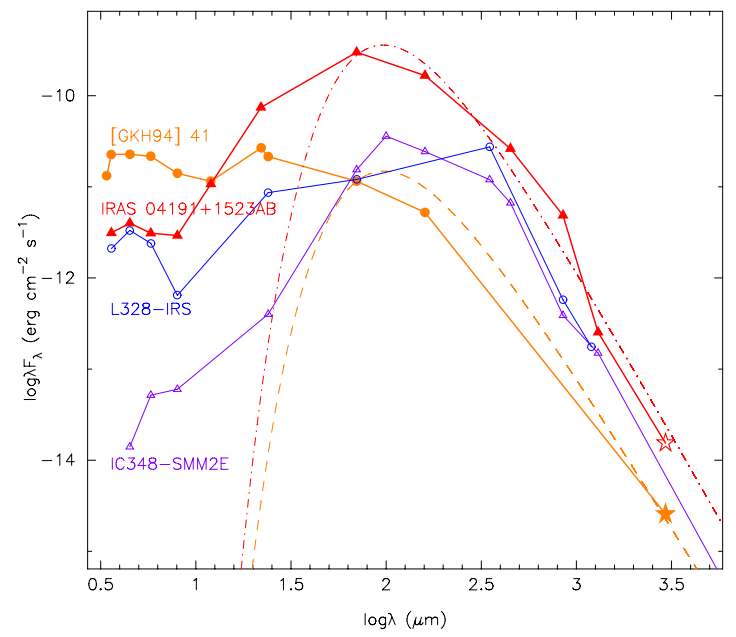

Fig. 4. SEDs of the class I VLM objects ([GKH94] 41: brown line, IRAS $04191+1523 \mathrm{AB}$ : red line) and previously reported class 0 protoBDs: L328-IRS Lee et al. (2013; blue line) and IC348-SMM2E Palau et al. (2014; violet line). For [GKH94] 41 and IRAS 04191+1523AB, infrared to submm data are taken from Bulger et al. (2014) and references therein. The $\mathrm{mm}$ data measured in this paper are indicated by the open and solid stars for IRAS 04191+1523AB and [GKH94] 41, respectively. The brown dashed line shows the best fit for a modified blackbody of the dust envelope of [GKH94] 41 and the red dash-dotted line for IRAS 04191+1523AB.

$A_{\mathrm{V}}=34.4$ (Bulger et al. 2014) and then derived a luminosity of $0.041 L_{\odot}$. The location of IRAS $04191+1523 \mathrm{~B}$ in the luminosity versus temperature diagram (Fig. 3) also indicates that the mass (including the uncertainties) of the central source is below $\sim 0.09 M_{\odot}$, which is consistent with the mass range derived from its spectral type. The upper limit to the current mass of IRAS $04191+1523 \mathrm{~B}$ is therefore in the range of 31-97 $M_{\mathrm{J}}$.

The fluxes at infrared and submm wavelengths are unresolved for components A and B. We thus used fluxes from $70 \mu \mathrm{m}$ to $\mathrm{mm}$ wavelengths (Table 3 ) from the two components to estimate the total mass of the envelopes. We applied the same method as for [GKH94] 41. The best fit (Fig. 4) gives $T_{\mathrm{d}}=$ $32 \mathrm{~K}, M_{\mathrm{env}}=33 M_{\mathrm{J}}$, and $\beta=0.7$ for IRAS $04191+1523 \mathrm{AB}$. The envelope mass of each component could also be estimated directly from fluxes at $\mathrm{mm}$ wavelengths. If we take $\beta=1.4$ (Chandler et al. 1998) and $T_{\mathrm{d}}=32 \mathrm{~K}$, we obtain the envelope masses of about $31 M_{\mathrm{J}}$ and $18 M_{\mathrm{J}}$ for IRAS $04191+1523 \mathrm{~A}$ and IRAS $04191+1523 \mathrm{~B}$, respectively. The total mass is now higher by a factor of $\sim 1.5$ than the value estimated from the fitting.
With the current mass of $57 M_{\mathrm{J}}$ and the envelope mass of $18 M_{\mathrm{J}}$, the upper limit to the final mass of IRAS $04191+1523 \mathrm{~B}$ is thus $75 M_{\mathrm{J}}$. If we include the uncertainty in its spectral type, the upper limit is $75_{-26}^{+40} M_{\mathrm{J}}$. This upper limit also indicates that IRAS $04191+1523 B^{-26}$ will likely become a BD or a VLM star with a mass just above the substellar limit.

\section{Conclusion}

We presented the detection of envelopes associated with the two class I VLM objects [GKH94] 41 and IRAS 04191+1523B. Our estimated final masses demonstrate that [GKH94] 41 and IRAS $04191+1523$ B will likely become BDs or VLM stars. The existence of these class I VLM objects strongly supports the scenario that BDs and VLM stars have the same formation processes as low-mass stars.

Acknowledgements. This research is funded by Vietnam National Foundation for Science and Technology Development (NAFOSTED) under grant number 103.08-2013.21. We would like to thank the referee for useful comments that significantly improved the paper. Support for CARMA construction was derived from the Gordon and Betty Moore Foundation, the Kenneth T. and Eileen L. Norris Foundation, the James S. McDonnell Foundation, the Associates of the California Institute of Technology, the University of Chicago, the states of California, Illinois, and Maryland, and the National Science Foundation. Ongoing CARMA development and operations are supported by the National Science Foundation under a cooperative agreement, and by the CARMA partner universities.

\section{References}

André, P., Ward-Thompson, D., \& Greaves, J. 2012, Science, 337, 69 Baraffe, I., Chabrier, G., Allard, F., \& Hauschildt, P. H. 1998, A\&A, 337, 403 Baraffe, I., Chabrier, G., Allard, F., \& Hauschildt, P. H. 2002, A\&A, 382, 563 Bate, M. R., Bonnell, I. A., \& Bromm, V. 2002, MNRAS, 332, L65 Bonnell, I. A., Clark, P., \& Bate, M.R. 2008, MNRAS, 389, 1556

Bourke, T. L., Myers, P. C., Evans II, N. J., et al. 2006, ApJ, 649, L37 Bulger, J., Patience, J., Ward-Duong, K., et al. 2014, A\&A, 570, A29 Chabrier, G., Baraffe, I., Allard, F., \& Hauschildt, P. H. 2000, ApJ, 542, 464 Chandler, C. J., Barsony, M., \& Moore, T. J. T. 1998, MNRAS, 299, 789 Duchêne, G., Bouvier, J., Bontemps, S., André, P., \& Motte, F. 2004, A\&A, 427, 651

Filippazzo, J. C., Rice, E. L., Faherty, J., et al. 2015, ApJ, 810, 158

Enoch, M. L., Evans, N. J. II, Sargent, A. I., \& Glenn, J. 2009, ApJ, 692, 973

Francesco, J. D., Johnstone, D., Kirk, H., MacKenzie, T., \& Ledwosinska, E. 2008, ApJS, 175, 277

Furlan, E., Luhman, K. L., Espaillat, C., et al. 2011, ApJS, 195, 3

Gomez, M., Kenyon, S. J., \& Hartmann, L. 1994, AJ, 107, 1850

Harsono, D., Jørgensen, J. K., van Dishoeck, E. F., et al. 2014, A\&A, 562, A77 Lee, C.-F. 2011, ApJ, 741, 62

Lee, C. W., Kim, M.-R., Kim, G., Saito, M., Myers, P. C., \& Kurono, Y. 2013, ApJ, 777, 50

Luhman, K. L. 2012, ARA\&A, 50, 65

Luhman, K. L., Stauffer, J. R., Muench, A. A., et al. 2003, ApJ, 593, 1093

Luhman, K. L., Mamajek, E. E., Allen, P. R., \& Cruz, K. L. 2009, ApJ, 703, 399

Luhman, K. L., Allen, P. R., Espaillat, C., Hartmann, L., \& Calvet, N. 2010, ApJS, 186, 111

Mathis, J. S. 1990, ARA\&A, 28, 37

Morata, O., Palau, A., González, R. F., et al. 2015, ApJ, 807, 55

Motte, F., \& André, P. 2001, A\&A, 365, 440

Padoan, P., \& Nordlund, ̊. 2004, ApJ, 617, 559

Palau, A., Zapata, L. A., Rodríguez, L. F., et al. 2014, MNRAS, 444, 833

Phan-Bao, N., Riaz, B., Lee, C.-F., et al. 2008, ApJ, 689, L141

Phan-Bao, N., Lee, C.-F., Ho, P. T. P., \& Tang, Y.-W. 2011, ApJ, 735, 14

Phan-Bao, N., Lee, C.-F., Ho, P. T. P., Dang-Duc, C., \& Li, D. 2014, ApJ, 795, 70

Rebolo, R., Zapatero Osorio, M. R., \& Martín, E. L. 1995, Nature, 377, 129

Ricci, L., Testi, L., Natta, A., et al. 2014, ApJ, 791, 20

Whitworth, A., Bate, M. R., \& Nordlund, A. 2007, in Protostars and Planets V, eds. B. Reipurth, D. Jewitt, \& K. Keil (Tucson, AZ: Univ. Arizona Press), 459 